\title{
Barreras en la comunicación percibidas por un grupo de mujeres histerectomizadas: Un desafío para entregar una educación apropiada
}

\author{
María Teresa Urrutia. PhD ${ }^{1 a}$, Paloma Beoriza ${ }^{2 b}$, Alejandra Araya PhD. ${ }^{1 a}$ \\ 1 Facultad de Enfermería, Universidad Andrés Bello. \\ 2 Escuela de Enfermería, Facultad de Medicina, Pontificia Universidad Católica de Chile.
}

\footnotetext{
${ }^{a}$ Enfermera-Matrona. ${ }^{b}$ Alumna de Enfermería.
}

Financiamiento para publicación: Fondo APA, Escuela de Enfermería Pontificia Universidad Católica de Chile.

\section{RESUMEN}

Antecedentes: La destreza comunicacional del profesional de la salud es reconocida como un aspecto crucial que facilita la educación a los pacientes. Objetivo: Describir las barreras que la mujer histerectomizada percibe en relación a la comunicación durante el proceso quirúrgico. Método: Estudio cualitativo realizado en 15 mujeres histerectomizadas. Para la recolección de los datos se utilizaron entrevistas en profundidad las que fueron analizadas con análisis de contenido. Resultados: Las mujeres identifican diferentes barreras tanto de ellas como de los profesionales que dificultan la comunicación y por ende la educación que ellas reciben en relación al proceso quirúrgico. Las barreras de los profesionales son el lenguaje técnico, falta de tiempo, enojo, contradicciones en las indicaciones y lejanía. Las barreras en las mujeres son la timidez, la vergüenza, la falta de confianza, el miedo y la conformidad. Conclusión: Los profesionales de salud deben enfocarse en desarrollar técnicas de comunicación que ayuden a los pacientes a disminuir las barreras y por ende mejorar los resultados en salud; deben entregar la información de forma clara y simple, verificando que lo comunicado ha sido entendido.

\section{PALABRAS CLAVES: Histerectomía, educación comunicación}

\section{SUMMARY}

Background: The communication skills of health care professional has been recognized as a crucial aspect that facilitates patient education. Aim: To describe the barriers that women perceive hysterectomy in relation to communication during the surgical procedure. Method: Qualitative study conducted in 15 hysterectomized women. Deep interview and content analysis were used. Results: Women identify different barriers from them and health care professionals, that difficult the communication and therefore the education that the women receive in relation to the surgical process. The professional's barriers are technical language, lack of time, anger, contradictions in the directions and distance. The barriers for women are shyness, shame, the lack of trust, fear and conformity. Conclusion: Health professionals should focus on developing communication techniques that help patients to reduce barriers and thus improve health outcomes; they must provide information in a clear and simple way, making sure that the statement has been understood.

KEY WORDS: Hysterectomy, education, communication 


\section{INTRODUCCIÓN}

La histerectomía es una de las cirugías más frecuentes después de la cesárea $(1,2)$ lo que hace que un gran número de mujeres se vean enfrentadas a este proceso quirúrgico (3). La presencia de síntomas de larga data (i.e. sangramiento, dolor abdominal, entre otros), producto de alguna patología benigna $(3,4)$ es una realidad frecuente en las mujeres histerectomizadas previo a esta cirugía $(4,5)$.

El hecho que la mayoría de las histerectomías son cirugías programadas $(3,4)$, permite desarrollar una intervención profesional acorde a las necesidades que la mujer presenta y evita, por lo tanto, que acuda a fuentes de información no profesionales para poder satisfacer sus dudas e inquietudes.

Si bien la histerectomía puede parecer una cirugía de rutina para el profesional, el significado que se le ha dado culturalmente al útero, hace que no sea considerada de rutina por la mujer. La asociación del órgano con conceptos tan potentes en nuestra cultura como son la maternidad, sexualidad y feminidad entre otros (6-8) sumado a los temores $(9,10)$ y el propio significado que la mujer le da a la cirugía, hacen de este proceso quirúrgico un desafío para los profesionales de salud en lo que respecta a la educación y por ende las vías de comunicación. La destreza comunicacional del profesional de la salud es reconocida como un aspecto crucial que facilita la educación a los pacientes $(11,12)$.

El objetivo de este estudio es describir la percepción que un grupo de mujeres histerectomizadas tiene en relación a las barreras comunicacionales, tanto de los profesionales como de ellas, durante su proceso quirúrgico.

\section{PACIENTES Y MÉTODO}

Estudio descriptivo cualitativo, realizado en 15 mujeres histerectomizadas mediante entrevistadas en profundidad. El grupo de estudio corresponde a una muestra por conveniencia de mujeres histerectomizadas por patología benigna durante los 6 meses previos a la recolección de los datos del Complejo Asistencial Dr. Sotero del Río (Santiago, Chile). La recolección de los datos fue realizada entre los meses de mayo a septiembre de 2010.

Cada entrevista estuvo direccionada por tres preguntas: 1) ¿Qué ha significado para usted que le hayan sacado el útero? 2) Como fue el apoyo/ educación que recibió? 3) Cual sería el apoyo/educación que le hubiese gustado recibir? El presente artículo analiza una de las dimensiones que emergieron frente a la segunda pregunta. Los resultados de la primera pregunta fueron publicados previamente (13).

Las entrevistas fueron realizadas en un lugar privado y grabadas, para posteriormente ser transcritas de manera textual. La duración promedio de cada entrevista fue de 40 minutos. Para el análisis de los datos se utilizó el análisis de contenido según Krippendorf (14). Los escritos fueron analizados por 4 investigadores de manera separada. Cada investigador propuso los significados y las dimensiones encontradas de manera independiente, discutiéndose cada propuesta hasta llegar a un consenso. Para evaluar la credibilidad de los análisis y asegurar la validez descriptiva, se usaron los criterios de Creswell (15). Primero: se utilizó el chequeo de los resultados obtenidos entre las mujeres, después de ser analizadas las entrevistas en profundidad, se realizaron grupos focales para corroborar la validez de los datos obtenidos en las entrevistas. Segundo: se incorporó una descripción detallada de los relatos en cada uno de los significados y dimensiones encontradas, de manera que el lector pudiera determinar su utilidad y aplicabilidad de los resultados de este estudio. Finalmente, el investigador principal examinó los resultados con 3 investigadores expertos en el área, los cuales consideraron válidos el análisis de datos.

Esta investigación contó con la aprobación de los Comités de Ética de la Escuela de Enfermería de la Pontificia Universidad Católica de Chile y del Servicio de Salud Metropolitano Sur Oriente.

\section{RESULTADOS}

La edad de las mujeres tiene un rango entre 35 a 73 años. La escolaridad de 3 a 17 años. Del total de mujeres: 11 son casadas, 3 solteras y 1 viuda. De aquellas que tienen pareja, el periodo de convivencia tiene un rango entre 14 y 50 años.

La causa más frecuente de HT fue la miomatosis uterina (9 casos). La vía de abordaje de la cirugía fue en 6 casos abdominal, 8 vaginal y en un caso indeterminado, dado que la usuaria afirma que ella aún conserva su útero y que no fue histerectomizada. En 7 casos hubo ooforectomía asociada a la histerectomía.

Las mujeres identifican diversas barreras que limitan la comprensión con los profesionales del área de la salud y que se transforman en barreras para lograr una comunicación efectiva entre las mujeres y los profesionales. Al categorizarlas se identifican limitaciones por parte de los profesionales y otras que evidencian la dificultad de las mujeres para relacionarse con el equipo de salud (Tabla I).

Barreras por parte del profesional: En relación al profesional de la salud, una de las barreras identificadas es el lenguaje técnico con el cual ellos se comunican, el cual dificulta la comprensión por parte de la mujer.

Mujer: "Hablan en términos médicos que uno no entiende $y .$. hablan entre ellos, entonces uno escucha no más y que más hace?,... que mas, que más hago yo? ...con términos médicos que nadie entiende en realidad". 


\section{Tabla I \\ BARRERAS EN LA COMUNICACIÓN PERCIBIDAS POR LAS MUJERES DURANTE EL PROCESO QUIRÚRGICO}

Del profesional de salud:
\[ \begin{array}{l}\text { Lenguaje técnico } \\ \text { Falta de tiempo } \\ \text { Percepción de enojo } \\ \text { Indiferencia } \\ \text { Contradicciones en la información } \\ \text { Lejanía }\end{array} \]

De la mujer:

Timidez
Vergüenza
Falta de confianza
Miedo
Conformidad

La falta de tiempo es otra de las barreras que las mujeres perciben por parte de los profesionales, y que les impide la posibilidad de conversar con ellos.

Mujer: "Porque ellos andan muy apurados, andan muy rápido, le miran la ficha a uno, ¿usted es la fulana de tal? sí doctor, ¿cómo se siente? bien, ¿le duele algo? no, ¿le hicieron aseo? si, ¿ha comido bien? si. Y le preguntan todas esas cosas, son muy gentiles, muy agradables, pero andan así... yummm... pasan rápido, uno no puede ponerse, no se puede sentar a conversar con los médicos".

El enojo por parte del personal de salud y la indiferencia son otras barreras percibidas por las mujeres.

Mujer: "Llegaba otra persona media cascarrabias y yo estaba con las sondas, necesitaba cosas, que me vieran, entonces había que estar con harta delicadeza, porque andaba como media enojada".

Mujer: "Es bueno que a uno se le informe y se le tome en cuenta lo que uno les dice... porque ...es como que uno habla y no se le escucha".

Las contradicciones en las indicaciones del personal de salud es otra barrera que la mujer identifica al momento de ser atendida.

Mujer: "Una enfermera da una orden otra enfermera da otra, entonces no sabe a quién hacerle caso y si uno le hace caso a una la otra se molesta. Por ejemplo lo de las medias, yo no sabía... yo llegué del pabellón y una niña me las sacó, después vino otra enfermera se enojó porque me las habían sacado y yo le dije yo acato las órdenes que dicen ustedes".

La lejanía o frialdad en la atención es otra de las barreras que las mujeres señalan marca una distancia entre ellas y el personal de salud.

Mujer: "Porque es todo tan frío... uno llega al hospital, lo ve una persona, la opera otra... estás en una sala con 6 personas más que no tienen el mismo problema tuyo, que ni siquiera los puedes conversar... te revisan todos los días físicamente cómo estás, pero nadie te pregunta nada más... Entonces, yo creo que es como muy frío todo... para una persona que sabe ... de qué se trata, no va a sentir mayormente ese proceso, pero para una persona que no tiene idea, estar ahí, que te vean la herida y sería todo... no sé, yo lo encuentro demasiado frío"

Barreras por parte de la mujer: Dentro de las barreras que las mujeres identifican en ellas misma se encuentran la timidez y la vergüenza.

Mujer: "Es que a veces hay personas tímidas, aunque no parezca... uno estando enferma es tímida y necesita que la ayuden, no sé, en ese momento que se siente como débil".

Mujer: "Vergüenza, vergüenza me daba... mucha vergüenza, ¿pero qué iba a hacer?, además que ellos ... son médicos y están acostumbrados a ver tanta operación y tanta cosa... que a ellos no les llama la atención me imagino, pero igual... igual yo sentía mucha vergüenza".

La falta de seguridad o "confianza" es otra de las barreras identificadas por las mujeres.

Mujer: "Lo atribuyo a no haber conocido al médico, porque cuando uno conoce al médico tiene como esa... el médico te dice estás bien, qué sé yo, entonces está como la como la confianza, aunque uno lo haya visto una vez, pero está la confianza".

El miedo es otra barrera que la mujer refiere la aleja del profesional y le impide resolver sus dudas.

Mujer: "Por miedo no pregunto. Porque el doctor va a decir "¿oye ésta a qué vino?, ¿cómo no va a saber a lo que vino?". Yo pensé que el doctor se podía enojar conmigo por tantas preguntas que uno a veces le hace"

La conformidad es otra causa por la cual la mujer no pregunta o resuelve dudas.

Mujer: “...no puedo pedir más... del hospital uno no puede pedir demasiado...porque... no sé, es público, es pobre, no puede pedir demasiado uno, entonces tiene que conformarse con lo que hay no más".

\section{DISCUSIÓN}

Es importante mencionar la escasez de publicaciones respecto a la educación que reciben las mujeres sometidas a una histerectomía, asimismo sobre las barreras o dificultades que pueden presentar tanto ellas como el equipo de salud. Sin embargo, ha sido descrita previamente la entrega de información insuficiente a las pacientes acerca de la cirugía, la anatomía femenina (16) y los efectos post-quirúrgicos (17).

No existen publicaciones que refieran las barreras comunicacionales de los profesionales y/o pacientes que se someten a una histerectomía, 
sin embargo existen estudios que mencionan las dificultades en la comunicación y/o educación en pacientes con otras patologías. En cuanto a las barreras que pueden presentar los equipos de salud se menciona el uso de jerga médica o lenguaje técnico con el que no se encuentran familiarizados los usuarios y el que no es explicado con claridad (18), coincidiendo con los resultados de este estudio. Por otro lado, se describe la entrega de mensajes poco claros e inadecuados como una causa por la que los pacientes no recibían una educación adecuada respecto a diagnósticos, pronósticos o cuidados post hospitalización (18). Junto con lo anterior, se habla del uso de expresiones médicas sin una explicación o comprensión por parte del usuario como un factor influyente en la aparición de angustia y ansiedad por parte del paciente (18). Cabe señalar que una buena educación a las mujeres sometidas a una histerectomía se asocia a una reducción de la ansiedad y experiencias de dolor (19-22).

Se hace un especial énfasis en la importancia de entregar explicaciones claras a los pacientes, particularmente en brindarles asistencia para que realicen una elección de tratamiento informada (23). Si la información no es entregada a los pacientes, estos pueden llegar a obtener sus propias conclusiones según la conducta no verbal de los profesionales de la salud (23). Lo mencionado anteriormente podría explicar los sentimientos percibidos por las usuarias en este estudio.

Cabe señalar que la frustración que las mujeres perciben por la falta de información, también ha sido documentada en relación a los profesionales. Las preguntas reiteradas por parte de los usuarios, quienes están confundidos o que no han comprendido del todo la información, generan en los profesionales un efecto que provoca resentimiento en el equipo de salud, además de frustración (18). Lo que podría afectar de manera negativa el proceso de aprendizaje de los usuarios al ser atendidos por personas con sentimientos negativos hacia el paciente que no logra comprender la información que se le entrega. Además, podría influir la percepción que el profesional tiene de sus propias habilidades y capacidades para entregar una atención de salud de calidad y centrada en los usuarios.

Otra de las barreras que arroja este estudio se relaciona con la contradicción en las indicaciones del personal de salud. La comunicación efectiva entre los profesionales de salud, se asocia con una mejoría de los resultados de los cuidados de los usuarios, de la satisfacción y retención de los profesionales y en algunos casos la disminución de los costos en salud(18). Además, genera una mejoría en cuanto la adherencia a las recomendaciones de tratamiento, bienestar psicológico y emocional y eleva la satisfacción de los pacientes hacia su proveedor de salud (18). Asimismo, se describe que la falta de una comunicación efectiva entre los funcionarios de la salud provoca confusión y una pérdida de confianza por parte de los usuarios hacia los miembros del equipo (18).

La lejanía es considerada como otra de las barreras de los profesionales para establecer una comunicación efectiva. La falta de empatía es manifestada en un comportamiento no verbal frío, mecánico, indiferente o negativo por parte de los funcionarios. En muchos casos este comportamiento es percibido como enojo o impaciencia hacia el usuario, lo que probablemente incrementa las barreras de los pacientes para comunicarse con su equipo de atención de salud (18).

Las pacientes reconocen como dificultades la timidez y vergüenza, la falta de seguridad o confianza, el miedo y la conformidad en el momento de interactuar con los profesionales.

En relación a las barreras por parte de las mujeres, se ha establecido una relación entre la vergüenza y timidez con una baja literacidad de los pacientes, además de la posibilidad de experimentar discriminación $(24,25)$. Por lo anterior es vital proveer un ambiente adecuado a las mujeres para que se sientan capaces de revelar abiertamente su necesidad de asistencia o confusión respecto a la información recibida (18). Cabe señalar que incluso aquellos individuos que poseen literacidad adecuada en salud pueden igualmente encontrar el ambiente médico amenazador y tener problemas para comprender la información que están recibiendo, situación que se acentúa si se encuentran enfermos, con dolor y/o preocupados sobre los efectos de su medicación (18).

\section{CONCLUSIÓN}

Los profesionales de salud deben enfocarse en desarrollar técnicas de comunicación que ayuden a los pacientes a disminuir las barreras y mejorar los resultados en salud; deben entregar la información de forma clara y simple, verificando que lo comunicado ha sido entendido. La comprensión de la información entregada, y la capacidad de autocuidado de las mujeres debe ser considerado un derecho, el cual todo profesional de salud debe proteger como también los proveedores de la atención de salud.

\section{REFERENCIAS}

1. Garry R, Fountain J, Mason S, Napp V, Brown J, Hawe $\mathrm{J}$, et al. The eVALuate study: two parallel randomised trials, one comparing laparoscopic with abdominal hysterectomy, the other comparing laparoscopic with vaginal hysterectomy. BMJ 2004;328(7432):129-33.

2. Sankaran S, Manyonda IT. Medical management of fibroids. Best Pract Res Clin Obstet Gynaecol 2008;22(4):655-76.

3. Flory N, Bissonnette F, Binik YM. Psychosocial effects of hysterectomy: literature review. J Psychosomatic Res 2005;59(3):117-29. 
4. Sabry M, Al-Hendy A. Medical treatment of uterine leiomyoma. Reprod Sci 2012;19(4):339-53.

5. Zimmermann A, Bernuit D, Gerlinger C, et al. Prevalence, symptoms and management of uterine fibroids: an international internet-based survey of 21,746 women. BMC Women's Health 2012;12:6

6. Lonnée-Hoffmann R1, Pinas. Effects of hysterectomy on sexual function. Curr Sex Health Rep 2014;6(4):244-51.

7. Bradford A, Meston C (2007) Sexual outcomes and satisfaction with hysterectomy: influence of patient education. J Sex Med 2007;4:106-14.

8. Reis N, Engin R, Ingec M, Bag B. A qualitative study: beliefs and attitudes of women undergoing abdominal hysterectomy in Turkey. Int J Gynecol Cancer 2008;18:921-8.

9. Gallicchio L, Harvey LA, Kjerulff KH. Fear of cancer among women undergoing hysterectomy for benign conditions. Psychosom Med 2005;67(3):420-4.

10. Wu SM, Chao Yu YM, Yang CF, Che HL. Decisionmaking tree for women considering hysterectomy. J Adv Nurs 2005;51(4):361-8.

11. Deledda G, Moretti F, Rimondini M, Zimmermann C. How patients want their doctor to communicate. A literature review on primary care patients' perspective. Patient Educ Couns 2013;90:297-306.

12. Bensing J, Rimondini M, Visser A. (2014). What patients want. Patient Educ Couns 2013;90(3):287-90.

13. Urrutia MT, Araya A, Flores C, Jara D, Silva S, Lira MJ. Histerectomía: la experiencia de no tener útero para un grupo de mujeres chilenas Rev Chil Obstet Ginecol 2013;78(4):262-8.

14. Krippendorf K. Content Analysis. An introduction to its methodology. Thousands Oaks: Sage Publications; 2004.
15. Creswell JW. Standards of validation and evaluation. Qualitative inquiry and research design: choosing among five approaches. 2 ed. Thousand Oaks, CA: Sage Publications; 2007. p. 201-21.

16. Wade J, Pletsch P, Morgan S, Menting S. Hysterectomy: what do women need and want to know? J Obstet Gynecol Neonatal Nurs 2000;29(1):33-42.

17. Corney R, Everett $\mathrm{H}$, Howells $A$, Crowther $M$. The care of patients undergoing surgery for gynaecological cancer: the need for information, emotional support and counselling. J Adv Nurs 1992;17(6):667-71.

18. Farahani M, Sahragard R, Carroll J, Mohammadi E. Communication barriers to patient education in cardiac inpatient care: A qualitative study of multiple perspectives. Inter J Nurs Pract 2011;17(3):322-8.

19. Cheung $L$, Callaghan $P$, Chang $A$. A controlled trial of psycho-educational interventions in preparing Chinese women for elective hysterectomy. Int J Nurs Stud 2003;4012)207-16.

20. Priya $P$, Roach $E$. Effect of pre-operative instruction on anxiety among women undergoing abdominal hysterectomy. Nurs J India 2013;104(6):245-8.

21. Callaghan $\mathrm{P}, \mathrm{Li} \mathrm{H}$. The effect of pre-operative psychological interventions on post-operative outcomes in Chinese women having an elective hysterectomy. $\mathrm{Br}$ J Health Psychol 2002;7(Pt2):247-52.

22. Araya A, Urrutia M. El proceso de ser histerectomizada: Aspectos educativos a considerar. Rev Chil Obstet Ginecol 2008;73(5):347-52.

23. Mody R. Overcoming barriers to delivering information to cancer patients. Br J Nurs 2003;12(21):1281-7.

24. Lambert V, Keogh D. Health literacy and its importance for effective communication Part 2. Nurs Child Young People 2014;26(4):32-6.

25. Oates D, Silliman R. Health Literacy: Improving patient understanding. Oncology 2009;23(4)376-9. 